\title{
Monoclinic angle, shear response, and minimum energy pathways of NiTiCu martensite phases from $a b$ initio calculations
}

\author{
Sam Bakhtiari ${ }^{1}$, Jefferson Zhe Liu ${ }^{2}$, Yinong Liu ${ }^{1 *}$, Hong Yang ${ }^{1}$ \\ ${ }^{1}$ Laboratory for Functional Materials, Department of Mechanical Engineering, The \\ University of Western Australia, 35 Stirling Highway, Perth, WA 6009, Australia \\ 2 Department of Mechanical Engineering, The University of Melbourne, Parkville, VIC 3010, \\ Australia \\ *Corresponding author email address: yinong.liu@uwa.edu.au, Phone: +61 86488 5664, \\ Fax: +61 864881024 .
}

\begin{abstract}
$\mathrm{Ti}_{50} \mathrm{Ni}_{50-\mathrm{x}} \mathrm{Cu}_{\mathrm{x}}$ alloys are observed to exhibit multiple martensitic transformations from $\mathrm{B} 2$ to an orthorhombic B19 and a monoclinic B19' phase. In addition, DFT calculations have predicted a B19" phase with a higher monoclinic angle as the thermodynamically stable ground state. This study investigated the effects of $\mathrm{Cu}$ content and shear stress on the monoclinic angles, phase stabilities of the various martensites, the minimum energy pathways, and the relative total energies among the phases in this pseudo-equiatomic $\mathrm{Ti}\left(\mathrm{Ni}_{50-\mathrm{x}} \mathrm{Cu}_{\mathrm{x}}\right)$ system. A new monoclinic phase (B19M) with a monoclinic angle lower than that of B19' was found at above a critical $\mathrm{Cu}$ content. This confirms the formation of an intermediate phase in the martensitic transformation sequence of the pseudo-equiatomic $\mathrm{Ti}\left(\mathrm{Ni}_{50-\mathrm{x}} \mathrm{Cu}_{\mathrm{x}}\right)$ system but contradicts the crystal structure of the experimentally observed phase. It was found that the monoclinic angles of both B19M and B19" decrease with increasing the magnitude of an opposing shear stress to their monoclinic distortion. At above certain critical values of the opposing shear stress, the B19M and B19" phases destabilise and transform to lower monoclinic angle phases. In addition, the evidence suggests that the experimentally observed monoclinic B19' phase is in fact a distorted B19" with a reduced monoclinic angle under an opposing shear stress. With the same argument, the experimentally reported B19 phase is a metastable phase formed under the effect of an opposing shear stress to the monoclinic distortion of В19м.
\end{abstract}

Keywords: Shape memory alloy; Martensitic phase transition; Ab initio calculations; Minimum energy pathway.

\section{Introduction}

NiTi shape memory alloys (SMAs) are known to exhibit unique thermomechanical properties including the shape memory effect and pseudoelasticity [1]. These properties originate from the thermoelastic martensitic transformations in these alloys [2, $\underline{3}]$. Owing to these remarkable properties, they have been used in many innovative designs, both in end product applications, such as low power actuators in automotive industry [4], wing morphing control in aerospace 
engineering [5] , miniature actuators in microelectromechanical systems (MEMS) [6], actuators in robotics [7] and biomedical devices [8], and in novel materials designs, such as intermetallic composites []ㅡ, functionally graded designs [10-13], and smart architectured materials [14]. To meet demands of different applications, their martensitic transformation characteristics and thermomechanical properties often need to be altered and controlled. One commonly used method to alter and control the properties is to add a third element into the binary NiTi, such as $\mathrm{Cu}, \mathrm{Nb}, \mathrm{Hf}$ and $\mathrm{Pd}$ [15-19]. Among them, Cu substitution for $\mathrm{Ni}$ is known to introduce an intermediate orthorhombic B19 phase in its martensitic transformation sequence, to narrow the transformation hysteresis [20], and to enhance thermal and mechanical cycling stability [21, 22]. These characteristics of ternary NiTiCu alloys make them attractive choices for many applications.

Solution treated equiatomic NiTi alloys are known to exhibit a B2 austenite and a B19' monoclinic martensite phase. Furthermore, density functional theory (DFT) calculations have also identified two other martensitic phases to B2 at $0 \mathrm{~K}$, including a monoclinic B19" and a base-centred orthorhombic (BCO) phase [23, 24]. The BCO phase is the ground state at $0 \mathrm{~K}$. Experimentally, those two phases are not observed though they are energetically stable phases in DFT calculations. In contrast, the experimentally observed $\mathrm{B} 19^{\prime}$ monoclinic martensite is thermodynamically unstable in DFT calculations [23, 25]. Stabilisation of B19' requires either an opposing shear stress to its monoclinic distortion [25] or a hydrostatic pressure [26, 27]. A recent DFT calculation suggests that inclusion of the thermal vibrational effect at a certain temperature may change the relative energy state order of the martensitic phases [28]. For example, a monoclinic structure with an angle of $\sim 101^{\circ}$, which corresponds to B19", is the ground state martensite instead of BCO at above $200 \mathrm{~K}$.

Complex transformation routes have been suggested among these phases of equiatomic NiTi. The experimentally observed martensitic transformation from B2 to B19' has been hypothesised to occur phenomenologically in two steps [1, 29]. The first step is B2 $\rightarrow$ B19. This transformation is associated with a volumetric lattice contraction and shuffling of the $\mathrm{Ni}$ and $\mathrm{Ti}$ atoms along the $[010]_{\mathrm{B} 19}$ direction. This produces the orthorhombic structure of the B19 phase. The second step is $\mathrm{B} 19 \rightarrow \mathrm{B} 19^{\prime}$. It involves a non-basal shear along [010] $]_{\mathrm{B} 19}$ direction and shuffling of $\mathrm{Ni}$ and $\mathrm{Ti}$ in the direction of $[001]_{\mathrm{B} 19}$. Further shear along $[010]_{\mathrm{B} 19}$ direction to reach $101.6^{\circ}$ and shuffle of $\mathrm{Ni}$ and $\mathrm{Ti}$ in the direction of [001] $]_{\mathrm{B} 19}$ produces B19", and then finally at $\sim 107^{\circ}$ monoclinic angle, BCO crystal structure forms. The B19 orthorhombic phase is imaginary for binary NiTi.

In $\mathrm{Ti}_{50} \mathrm{Ni}_{50-\mathrm{x}} \mathrm{Cu}_{\mathrm{x}}$ alloys when the $\mathrm{Cu}$ content exceeds 7.5 at\%, the original $\mathrm{B} 2 \leftrightarrow \mathrm{B} 19^{\prime}$ one-step transformation sequence has been observed to change to two steps, i.e., B2 $\leftrightarrow \mathrm{B} 19 \leftrightarrow \mathrm{B} 19^{\prime}[15$, $20,30,31]$. When $\mathrm{x} \geq 15$ at $\%, \mathrm{~B} 19^{\prime}$ vanishes and the transformation sequence becomes $\mathrm{B} 2 \leftrightarrow \mathrm{B} 19$ [32]. However, some recent studies have shown that the B19' phase can still be present in alloys of 16 and 20 at\% Cu contents [프, $\underline{34]}$.

Although the B19 phase has been well observed experimentally and the empirical criterion for its formation is established, a clear theoretical ground to explain the formation of the B19 phase and the change of the transformation sequence from B2 $\leftrightarrow \mathrm{B} 19^{\prime}$ to $\mathrm{B} 2 \leftrightarrow \mathrm{B} 19 \leftrightarrow \mathrm{B} 19^{\prime}$ and then to $\mathrm{B} 2 \leftrightarrow \mathrm{B} 19$ with increasing $\mathrm{Cu}$ content is lacking in the literature.

In this study, the effect of $\mathrm{Cu}$ content on the structures of the phases in the pseudo-equiatomic $\mathrm{Ti}\left(\mathrm{Ni}_{50-\mathrm{x}} \mathrm{Cu}_{\mathrm{x}}\right)$ system was investigated by means of density functional theory (DFT) calculation. Pure shear stresses were applied to study the stability of the martensitic phases under such conditions to establish fundamental understandings of the behaviour of the real alloy system. 


\section{Methodology}

The DFT simulations were carried out using Vienna $a b$ initio Simulations Package (VASP) [35]. The PBE exchange-correlation functional [36] and the projector-augmented method (PAW) [37] were employed to perform the simulations. All calculations applied an energy cutoff of $500 \mathrm{eV}$, an electronic energy convergence criterion of $1 \times 10^{-7} \mathrm{eV}$, and the $k$-point mesh density of at least $50 \mathrm{k}$-points per $\AA^{-1}$. The maximum force on each atom after relaxation was less than $5 \times 10^{-3} \mathrm{eV}^{-1}$ and the simulations were spin polarised. The minimum energy pathways (MEP) between fully relaxed structures were performed using the generalised solid-state nudge elastic band (G-SSNEB) method implemented in the VTST package [38]. The initial transition path between two fully relaxed structures was constructed by linearly interpolating the cell vectors and ionic positions for eight intermediate images. The method is capable of changing shape, size and ionic positions of intermediate images between two fully relaxed phases to find the energetically lowest possible states.

The size of the supercells for the most of the DFT calculations for the effects of pure shear and for the minimum energy pathways was 32 atoms, created from $2 \times 2 \times 2$ four-atom primitive unit cells. The $\mathrm{Cu}$ addition was achieved through random $\mathrm{Ni}$ atom substitution for each composition. Given the limited supercell size, only four $\mathrm{Cu}$ contents of $\mathrm{x}=0$, 3.125, 6.250, 9.375 and 12.500 at\% were investigated. For each Cu content, three different $\mathrm{Cu}$ doping patterns were calculated to avoid artefacts from doping patterns. In this study, the BCO phase is not considered due to the limited size of the employed supercells, which could easily break the high symmetry of the BCO phase. To validate the results of the $2 \times 2 \times 2$ supercell calculations, especially the formation of the new phase and the monoclinic angle of the martensite phases, $3 \times 3 \times 3$ supercells (containing 108 atoms) of special quasi-random structures (SQS) [39] were generated for different $\mathrm{Cu}$ contents. The SQS method mimics the correlation functions of a perfectly random structure and can approximate the random solid solution [39].

A geometry-controlled calculation was performed to determine the resistive and assistive shear stresses required to distort the monoclinic angle of a phase from tis relaxed state. The calculation was completed using the VASP automatic relaxation routine via optimisation of the lattice parameters and relaxation of ionic positions. Then the lattice parameters are optimised by reducing all stress components to $0.01 \mathrm{GPa}$ except the shear stress corresponding to the monoclinic angle distortion applied. This process allows a pure shear stress corresponding to the applied monoclinic distortion to be calculated. Fig. 1 shows the changes of the total energy and the lattice parameters of $\mathrm{B} 19^{\prime \prime}$ in $\mathrm{Ti}_{50} \mathrm{Ni}_{37.5} \mathrm{Cu}_{12.5}$ when the monoclinic angle is set at $95^{\circ}$, which is a $-3^{\circ}$ distortion from the $98^{\circ}$ at its relaxed state. The iterations were continued until the total energy convergence criterion of $10^{-7} \mathrm{eV} /$ atom is reached and the maximum force on each atom is below $5 \times 10^{-3} \mathrm{eV} \AA^{-1}$.

Using this approach, the shear stress thus calculated is a function of the monoclinic angle distortion from its relaxed state. The maximum shear stress (positive or negative) on monoclinic distortion (increase or decrease) of a monoclinic phase signifies the critical condition at which the phase becomes energetically unfavourable and beyond which the phase transforms to a new phase with a higher or lower monoclinic angle. These maximum stresses are thus the critical shear stresses for transformations. 


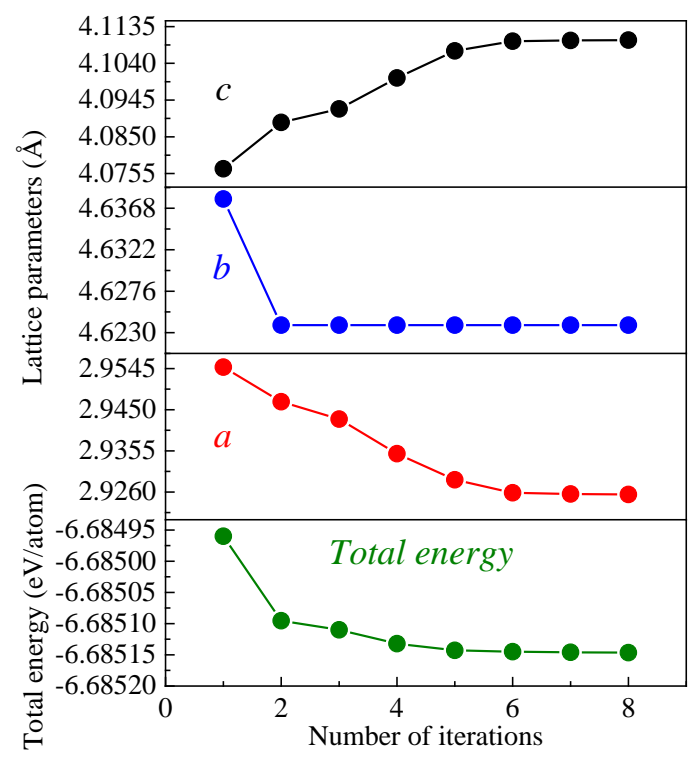

Figure 1. Optimisation of the total energy and the lattice parameters of B19" in Ti ${ }_{50} \mathrm{Ni}_{37.5 \mathrm{Cu}} \mathrm{Cu}_{2.5}$ at $95^{\circ}$ monoclinic angle, which is a $-3^{\circ}$ distortion from its relaxed state.

\section{Results and discussions}

Fig. 2 shows the effect of $\mathrm{Cu}$ addition on the monoclinic angles of the martensitic phases in the $\mathrm{Ti}(\mathrm{Ni}, \mathrm{Cu})$ pseudo-equiatomic system. It is seen that the monoclinic angle of B19" decreases with increasing $\mathrm{Cu}$ content. The monoclinic angle difference between the $2 \times 2 \times 2$ (circular blue data points) and $3 \times 3 \times 3$ (rectangular blue data points) supercells is negligible. The B19' phase is not a thermodynamically stable phase, thus it cannot be predicted by DFT calculation. The data shown in the figure are experimental data reported in the literature $[30,40]$. It is seen that the monoclinic angle of $\mathrm{B} 19^{\prime}$ also decreases with increasing $\mathrm{Cu}$ content. There is a clear difference between the monoclinic angles of B19" (calculated) and B19' (experimental) phases.

Our DFT calculations also revealed another monoclinic phase at above a critical $\mathrm{Cu}$ content, denoted B19 $\mathrm{M}$ in Fig. 2. To form the B19 $\mathrm{M}$ phase, a large enough (i.e. $1^{\circ}$ ) increase or decrease of monoclinic distortion can be applied to the relaxed B19 or B19" phases, respectively. Subsequent VASP automatic relaxation may stabilise the В19м phase. A small distortion reverts back to the fully relaxed distorted structure. Apparently, at below the critical Cu content, the distorted B19" transforms to B19 and vice versa. The critical $\mathrm{Cu}$ content for the stabilisation of the $\mathrm{B} 19 \mathrm{M}$ phase for $2 \times 2 \times 2$ and $3 \times 3 \times 3$ supercells are $\mathrm{x}=6.25$ at $\%$ and 9.25 at\%, respectively. The difference in the critical $\mathrm{Cu}$ content to stabilise the new phase can be attributed to the limitations of the smaller supercells, and the value obtained from the larger supercells is expected to better approximate the random solid solution condition. To further investigate the onset of the stabilisation of the B19м phase, a $4 \times 4 \times 4$ (256 atoms) SQS supercell with $x=9.375$ at\% was constructed. The increase of the size of the supercell did not affect the monoclinic angle or the onset of the stabilisation.

The presence of this new phase at above 9.25 at\% $\mathrm{Cu}$ in the DFT calculation coincides with the presence of the $\mathrm{B} 19$ phase at above $7.5 \mathrm{at} \% \mathrm{Cu}$ in experiment [15]. However, the monoclinic crystal structure of the $\mathrm{B} 19 \mathrm{M}$ phase predicted by the calculation contradicts the experimentally observed orthorhombic phase (B19). The monoclinic angle of this phase is below those of B19' (experimental) and B19" (calculated) phases. This phase is denoted B19M in recognition of its monoclinic lattice distortion. Its monoclinic angle is also calculated and 
found to decrease with increasing $\mathrm{Cu}$ content. Fig. 2 also presents the calculated B19 phase at different $\mathrm{Cu}$ contents. The data points shown in the figure for the B19 phase are the fully relaxed phases. No change to its orthorhombic angle is expected with changing the Cu content.

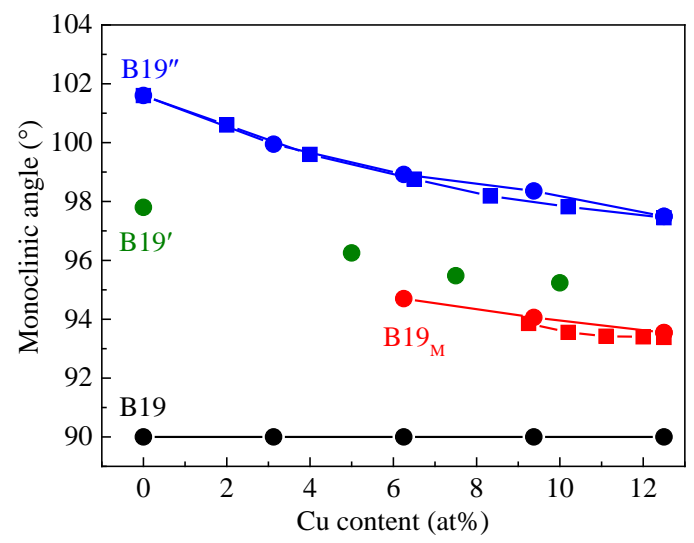

Figure 2. Effect of Cu content on the monoclinic angles of martensites in the pseudoequiatomic $\mathrm{Ti}(\mathrm{Ni} 50-\mathrm{xCux})$ system. The data for B19' are from experimental measurements $[\underline{30}, \underline{40}$. (The circular and rectangular data points are calculated from $2 \times 2 \times 2$ and $3 \times 3 \times 3$ supercells, respectively).

In DFT calculations, the B19' phase is known to be thermodynamically unstable, i.e., it does not exist except under the influences of external factors such as a resistive shear stress [25] (a shear stress opposing the monoclinic distortion of the phase) or a hydrostatic pressure [26, 27]. The resistive shear stress and hydrostatic pressure can be self-generated during the martensitic phase transformation because B2 $\rightarrow \mathrm{B} 19^{\prime \prime}$ (and also B2 $\rightarrow \mathrm{B} 19^{\prime}$ ) is associated with a monoclinic lattice distortion and a volume expansion. In our previous work [27], we revealed that the B19' structure can be stabilised at 2 GPa hydrostatic pressure without the presence of a resistive shear stress. Increase of the hydrostatic pressure reduces the monoclinic angle of the martensite phases and induces the $\mathrm{B} 19^{\prime \prime} \rightarrow \mathrm{B} 19^{\prime}$ transformation at $\sim 6.3 \mathrm{GPa}$ and the $\mathrm{BCO} \rightarrow \mathrm{B} 19^{\prime}$ transformation at $\sim 9 \mathrm{GPa}$ [27].

The B19' structure can also be achieved by applying a resistive shear stress to B19". Fig. 3 shows the effect of a resistive shear stress on the monoclinic angle, relative total energy, and phase stability of $\mathrm{B} 19^{\prime \prime}$ in the $\mathrm{Ti}_{50} \mathrm{Ni}_{50}$ alloy system (i.e., $\mathrm{x}=0 \mathrm{at} \% \mathrm{Cu}$ ). Fig. 3(a) shows the effect of resistive shear stress on the monoclinic angle and phase stability of B19". The calculation shows that the monoclinic angle of B19" decreases progressively with increasing the magnitude of the resistive shear stress and that at -0.85 GPa shear stress the monoclinic angle is reduced to $97.8^{\circ}$, the value measured experimentally for $\mathrm{B} 19^{\prime}$. This suggests that the B19' phase may in fact be a distorted B19" at -0.85 GPa opposing shear stress. Further increase of the opposing shear stress destabilises B19" towards the orthorhombic B19 (the dash line in the figure). The critical point of the destabilisation is at $95.5^{\circ}$ monoclinic angle and $-1.17 \mathrm{GPa}$ shear stress. The critical monoclinic angle and resistive shear stress are in a good agreement with the previous calculation by Wagner and Windl [25]. The only difference between this work and their analysis is the initial crystal structure. In this work we applied the opposing shear stress to B19", and they started from the BCO structure. Fig. 3(b) shows the effect of monoclinic angle, which is influenced by the resistive shear stress, on the relative total energy of B19". The total energy of B19 relative to that of B19" is also shown in the figure. The relative total energy of B19" increases with decreasing the monoclinic angle (caused by the increase of the resistive shear stress) to $95.5^{\circ}$, at which point it destabilises into B19. The B19 phase is at a higher relative total energy position than B19". The monoclinic angle of the 
experimentally observed B19' phase is also indicated in the figure. It is apparent that the B19' phase is not a local minimum under shear stress.
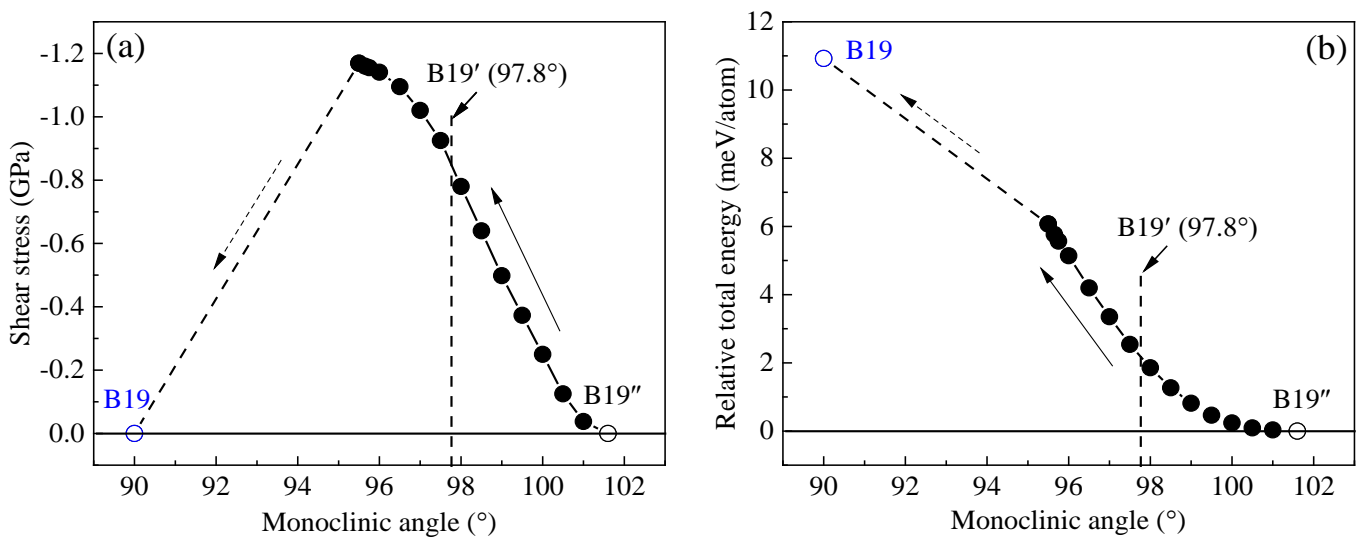

Figure 3. $\mathrm{Ti}_{50} \mathrm{Ni}_{50}$ alloy system. (a) effect of resistive shear stress on the monoclinic angle and phase stability of B19"; (b) effect of resistive shear stress on the relative total energy of B19" as reflected by the variation of the monoclinic angle.

Similar to the equiatomic NiTi case, shear stresses can be applied to the martensitic phases of the pseudo-equiatomic $\mathrm{Ti}\left(\mathrm{Ni}_{50-\mathrm{x}} \mathrm{Cu}_{\mathrm{x}}\right)$ system to achieve the experimentally reported $\mathrm{B} 19^{\prime}$ and also to reveal the shear response of B19м. Fig. 4 shows the effect of a resistive shear stress on B19M and B19" in the Ti ${ }_{50} \mathrm{Ni}_{43.75} \mathrm{Cu}_{6.25}$ alloy system. Fig. 4(a) shows the effect of the shear stress on the monoclinic angles and phase stabilities of the two phases. The monoclinic angle of B19" decreases with increasing the opposing shear stress. At -0.75 GPa shear stress, the monoclinic angle is reduced to $95.5^{\circ}$ and B19" destabilises towards B19M. Comparing with the case for equiatomic NiTi, the Cu substitution for Ni decreases the critical shear stress required to destabilise B19". A linear interpolation between the experimentally reported monoclinic angles of B19' (see Fig. 2) at 5 and 7.5 at $\%$ Cu reveals a monoclinic angle of $95.9^{\circ}$ for ${\mathrm{B} 19^{\prime}}^{\prime}$ at $6.25 \mathrm{at} \% \mathrm{Cu}$, which is indicated in the figure. It is very close to the calculated critical destabilisation monoclinic angle of B19" under the influence of the opposing shear stress.
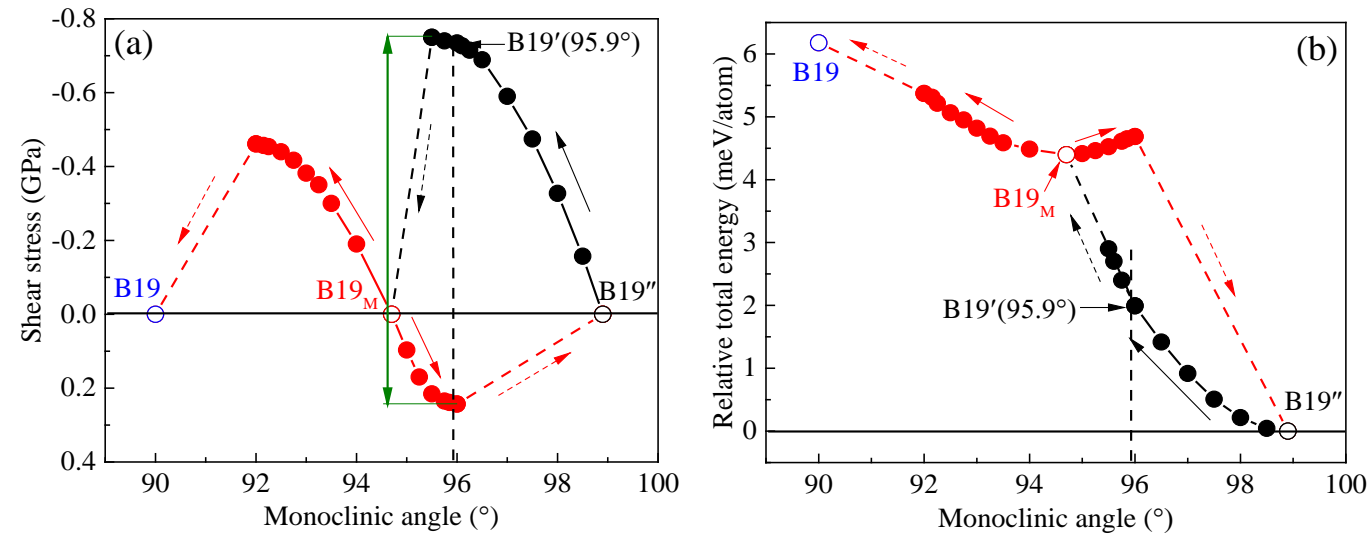

Figure 4. $\mathrm{Ti}_{50} \mathrm{Ni}_{43.5} \mathrm{Cu}_{6.25}$ alloy system. (a) effect of shear stress on the monoclinic angle and phase stability of B19M and B19"; (b) effect of shear stress on the relative total energy of B19 $\mathrm{M}$ and B19" as reflected by the variation of the monoclinic angle.

The opposing shear stress is also applied to the B19M phase, as seen in Fig. 4(a). Similar to B19", the monoclinic angle of B19M decreases progressively with increasing the opposing shear stress. It destabilises into B19 at the critical monoclinic angle of $92^{\circ}$ at $-0.46 \mathrm{GPa}$ shear 
stress. For comparison, an assistive shear stress, which is in the direction of the monoclinic distortion, is also applied to the $\mathrm{B} 19 \mathrm{M}$ phase. In contrary with the opposing shear stress, the monoclinic angle of $\mathrm{B} 19 \mathrm{M}$ increases progressively with increasing the assistive shear stress. At $+0.24 \mathrm{GPa}$ shear stress, the monoclinic angle is increased to $96^{\circ}$ and the $\mathrm{B} 19 \mathrm{M}$ phase is destabilised to B19". The shear stress hysteresis for the B19 $\mathrm{M} \leftrightarrow \mathrm{B} 19^{\prime \prime}$ transformation is 0.99 GPa as indicated by the double headed arrow in Fig. 4(a).

Fig. 4(b) shows the effect of shear stress, as reflected by the monoclinic angle change, on the relative total energies of B19" and B19 M phases. It is seen that the relative total energy of B19" decreases with decreasing the monoclinic angle (i.e., increasing the resistive shear stress), till the critical point at $95.5^{\circ}$ when B19" becomes unstable and transforms to B19M, as indicated by the dashed line. In comparison, the relative total energy of $\mathrm{B} 19 \mathrm{M}$ increases with both increasing and decreasing the monoclinic angle (corresponding to the increase of both an assistive and a resistive shear stress, respectively). This means that $\mathrm{B} 19_{\mathrm{M}}$ is a local shear minimum. The $\mathrm{B} 19_{\mathrm{M}}$ phase becomes unstable when the monoclinic angle is reduced to $92^{\circ}$ and transforms to B19 under the influence of a resistive shear stress, or when the monoclinic angle is increased to $96^{\circ}$ and transforms to B19" under the influence of an assisting shear stress. The shear energy barrier of $\mathrm{B} 19_{\mathrm{M}} \rightarrow \mathrm{B} 19^{\prime \prime}$ phase transformation is $\sim 0.3 \mathrm{meV} / \mathrm{atom}$. The total energy of B19 relative to that of B19" is also shown in the figure. It is seen that the relative total energy of B19 is above those of both В19м and B19". Also in comparison with the relative total energy of B19 in the equiatomic NiTi alloy, the addition of $\mathrm{Cu}$ has lowered the total energy of B19 relative to that of B19".

Similar calculations have also been performed for $\mathrm{x}=12.5$ at\%. Fig. 5 shows the effects of shear stress on the B19M and B19" phases in the $\mathrm{Ti}_{50} \mathrm{Ni}_{37.5} \mathrm{Cu}_{12.5}$ alloy. Fig. 5(a) shows the effects of shear stress on the monoclinic angles and phase stabilities of B19 $\mathrm{M}$ and B19". Similar to the previous two cases, the monoclinic angle of B19" decreases with increasing the opposing shear stress. At -0.72 GPa shear stress the monoclinic angle is reduced to $95^{\circ}$ and B19" becomes unstable and transforms to $\mathrm{B} 19 \mathrm{M}$. The values of the shear stress and monoclinic angle of the critical point for B19" $\rightarrow \mathrm{B} 19_{\mathrm{M}}$ are both slightly lower than those for the Ti ${ }_{50} \mathrm{Ni}_{43.5} \mathrm{Cu}_{6.25}$ alloy. The monoclinic angle of B19' corresponding to this alloy composition was estimated via linear extrapolation from the experimental data shown in Fig. 2 to be $95.1^{\circ}$, as indicate in the figure. It is very close to the calculated critical monoclinic angle of B19" under the influence of an opposing shear stress.
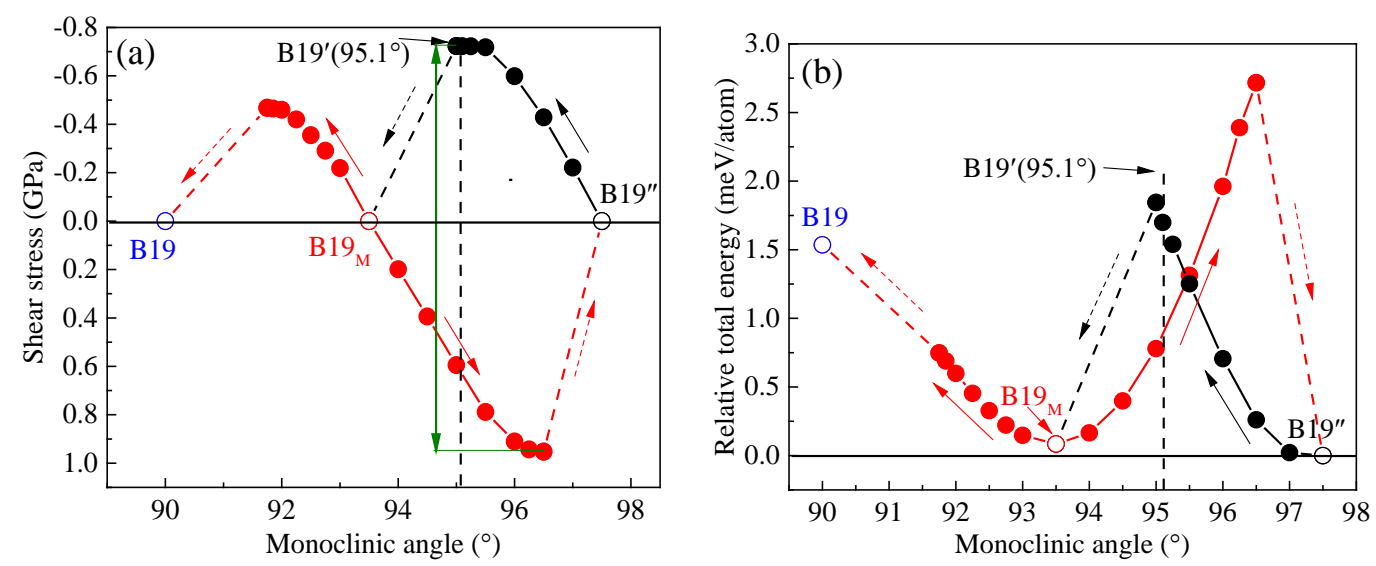

Figure 5. $\mathrm{Ti}_{50} \mathrm{Ni}_{37.5} \mathrm{Cu}_{12.5}$ alloy system. (a) effect of shear stress on the monoclinic angle and phase stability of B19M and B19"; (b) effect of shear stress on the relative total energy of B19 $\mathrm{M}$ and B19" as reflected by the variation of the monoclinic angle. 
The opposing shear stress is also applied to В19м phase. Similar to B19", the monoclinic angle of $\mathrm{B} 19 \mathrm{M}$ decreases progressively with increasing the opposing shear stress. The critical monoclinic angle is $91.75^{\circ}$ and the critical shear stress is $-0.45 \mathrm{GPa}$ for the $\mathrm{B} 19_{\mathrm{M}} \rightarrow \mathrm{B} 19$ transformation. When an assistive shear stress is applied to $\mathrm{B} 19 \mathrm{M}$, the monoclinic angle increases progressively till $96.5^{\circ}$ at $+0.95 \mathrm{GPa}$ shear stress when it becomes unstable and transforms to B19". The shear stress hysteresis for the B19 $\leftrightarrow$ B19" transformation is 1.67 $\mathrm{GPa}$, which is much higher than that for the Ti50Ni43.5Cu6.25 alloy.

Fig. 5(b) shows the effect of shear stress, as reflected by the variation of the monoclinic angle, on the relative total energies of the martensite phases. The relative total energy of B19" decreases with decreasing the monoclinic angle (increasing the opposing shear stress). There is a shear energy barrier of $1.84 \mathrm{meV} /$ atom for $\mathrm{B} 19^{\prime \prime} \rightarrow \mathrm{B} 19_{\mathrm{M}}$. B19M is still a local shear minimum. The reverse $\mathrm{B} 19_{\mathrm{M}} \rightarrow \mathrm{B} 19^{\prime \prime}$ transformation may occur by the application of an assisting shear stress (a shear stress in the direction of the monoclinic distortion, thus increase of the monoclinic angle). The shear energy barrier of the $\mathrm{B} 19_{\mathrm{M}} \rightarrow \mathrm{B} 19^{\prime \prime}$ transformation is $\sim 2.72$ meV/atom, which is higher than that for the $\mathrm{Ti}_{50} \mathrm{Ni}_{43.5 \mathrm{Cu} 6.25}$ alloy. This implies that $\mathrm{B} 19 \mathrm{M}$ is further stabilised relative to B19" at higher $\mathrm{Cu}$ contents.

Values of the shear stresses and the monoclinic angles at the critical points for the $B 19_{M} \rightarrow B 19$, $\mathrm{B} 19_{\mathrm{M}} \rightarrow \mathrm{B} 19^{\prime \prime}$, and $\mathrm{B} 19^{\prime \prime} \rightarrow \mathrm{B} 19_{\mathrm{M}}$ phase transformations as determined from the above calculations are summarised in Table 1. Also derived from these values are the shear stress hysteresis $(\Delta \tau)$ for B19" $\leftrightarrow \mathrm{B} 19_{\mathrm{M}}$. It is seen that by increasing the $\mathrm{Cu}$ content in the pseudoequiatomic $\mathrm{Ti}\left(\mathrm{Ni}_{50-\mathrm{x}} \mathrm{Cu}_{\mathrm{x}}\right)$ system, the critical resistive shear stress required to destabilise B19" phase reduces. It can also be seen that the critical resistive shear value to destabilise B19" is higher than that of $\mathrm{B} 19 \mathrm{M}$. The increase of the $\mathrm{Cu}$ content also increases the shear hysteresis of the $\mathrm{B} 19_{\mathrm{M}} \leftrightarrow \mathrm{B} 19^{\prime \prime}$ transformation. The increase of the shear hysteresis can be interpreted as stabilisation of $\mathrm{B} 19 \mathrm{M}$ relative to B19".

Table 1. Summary of the calculated shear stresses, monoclinic angles, and shear hysteresis at the critical points for the $\mathrm{B} 19_{\mathrm{M}} \rightarrow \mathrm{B} 19, \mathrm{~B} 19_{\mathrm{M}} \rightarrow \mathrm{B} 19^{\prime \prime}$, and $\mathrm{B} 19^{\prime \prime} \rightarrow \mathrm{B} 19_{\mathrm{M}}$ phase

transformations.

\begin{tabular}{|c|c|c|c|c|c|c|c|}
\hline \multirow{2}{*}{ at\% $\mathrm{Cu}$} & \multicolumn{2}{|c|}{$\mathrm{B} 19_{\mathrm{M}} \rightarrow \mathrm{B} 19$} & \multicolumn{2}{c|}{$\mathrm{B} 19_{\mathrm{M}} \rightarrow \mathrm{B} 19^{\prime \prime}$} & \multicolumn{2}{c|}{$\mathrm{B} 19^{\prime \prime} \rightarrow \mathrm{B} 19_{\mathrm{M}}(\mathrm{B} 19)$} & $\mathrm{B} 19^{\prime \prime} \leftrightarrow \mathrm{B} 19_{\mathrm{M}}$ \\
\cline { 2 - 8 } & $\tau(\mathrm{GPa})$ & $\beta\left(^{\circ}\right)$ & $\tau(\mathrm{GPa})$ & $\beta\left(^{\circ}\right)$ & $\tau(\mathrm{GPa})$ & $\beta\left(^{\circ}\right)$ & $\Delta \tau(\mathrm{GPa})$ \\
\hline 0 & - & - & - & - & -1.17 & 95.5 & - \\
\hline 6.25 & -0.46 & 92 & +0.24 & 96 & -0.75 & 95.5 & +0.99 \\
\hline 12.5 & -0.45 & 91.75 & +0.95 & 96.5 & -0.72 & 95 & +1.67 \\
\hline
\end{tabular}

According to these findings, the theoretical thermal transformation sequence is expected to be $\mathrm{B} 2 \leftrightarrow \mathrm{B} 19 "$ (BCO phase is not considered in this discussion) at $\mathrm{Cu}<9.25$ at\% and is $\mathrm{B} 2 \leftrightarrow \mathrm{B} 19_{\mathrm{M}} \leftrightarrow \mathrm{B} 19 "$ at $\mathrm{Cu} \geq 9.25$ at\%, when under no external influences (e.g., shear stress or hydrostatic pressure). The DFT prediction of the appearance of the intermediate B19M phase at above 9.25 at\% Cu coincides with the experimental observation of the B19 phase [ $\underline{15}, \underline{20}$, 30, 31]. However, there exist a couple of discrepancies between the theoretical calculations and experimental observations. First, at below 9.25 at\% Cu, the predicted martensite is B19" whereas the experimentally observed martensite is B19', which has a smaller monoclinic angle. Secondly, $\mathrm{Cu} \geq 9.25$ at\%, the experimentally observed intermediate phase is B19, which has an orthorhombic structure, whereas the theoretically predicted phase is B19M, which has a monoclinic structure. 
The discrepancy between B19" and B19' has been rationalised here by the effect of the inherent resistive shear stress self-generated by the monoclinic distortion of the transformation itself. The evidence presented in Fig. 3(a) (similarly in Figs. 4(a) and 5(a)) also suggests that the B19' phase is a distorted B19" with a reduced monoclinic angle instead of a stable phase in its own right, since that it is not a local shear minimum but a passing state on the continuous curve of the monoclinic angle vs shear stress. This hypothesis also implicitly implies that the monoclinic angle of the experimentally observed B19' can vary depending on the magnitude of the local opposing shear stress. Considering the stiffness tensor of B19' martensite (taken also as for B19") calculated by Wagner and Windl [25] and its monoclinic shear distortion, the opposing shear stress generated within a rigid body can be estimated to be $\sim 3 \mathrm{GPa}$, apparently sufficient to reduce the monoclinic angle of B19" to that of the experimentally observed B19'.

By the same argument, the theoretically predicted monoclinic B19M is also expected to selfgenerate an opposing shear stress, which will also reduce its monoclinic distortion, as expressed in Figs. 4(a) and 5(a). The self-generated resistive shear stress may well be above the critical value required to destabilise B19м. This may lead to the experimentally observed orthorhombic B19 phase. This is also plausible in that the critical shear stress of $B 19_{M} \rightarrow B 19$ is significantly lower than that required for B19" $\rightarrow \mathrm{B} 19^{\prime}$.

Summarising the above analyses, it is believed that the experimentally observed B19' is in fact a distorted B19" with a reduced monoclinic angle and that the experimentally observed B19 is induced from B19M, with both process caused by the self-generated opposing shear stress associated with the monoclinic distortion of the martensites.

To study the stabilities of the B19, B19M and B19" phases and the possible transformation routes, the minimum energy pathways (MEPs) between them are established. Fig. 6 shows the MEPs for $\mathrm{B} 19 \leftrightarrow \mathrm{B} 19_{\mathrm{M}}$ and $\mathrm{B} 19_{\mathrm{M}} \leftrightarrow \mathrm{B} 19^{\prime \prime}$ phase transformations. Fig. 6(a) shows the $\mathrm{B} 19 \leftrightarrow \mathrm{B} 19_{\mathrm{M}}$ transformation at three different $\mathrm{Cu}$ contents. The B19M phase has a lower energy state than the $\mathrm{B} 19$ phase, and the $\mathrm{B} 19 \rightarrow \mathrm{B} 19_{\mathrm{M}}$ transformation is barrier-less, implying a spontaneous transformation and the instability of B19 (B19 is not a local minimum) under no external influences (e.g., shear stress and hydrostatic pressure). Fig. 6(b) shows the $\mathrm{B} 19 \mathrm{M} \leftrightarrow \mathrm{B} 19 "$ transformation at three different Cu contents. An energy barrier exists between the two phases for all the three cases, and its magnitude increases with increasing the $\mathrm{Cu}$ content. It is apparent that below the critical level of $\mathrm{Cu}$ content, the B19 ${ }_{M} \rightarrow \mathrm{B} 19^{\prime \prime}$ is barrierless, thus the B19M structure transforms to B19" (the ground state). The energy barrier implies that $\mathrm{B} 19_{\mathrm{M}}$ can be a local minimum, which can be the reason for the formation of $\mathrm{B} 19_{\mathrm{M}}$. It is also seen that the energy level of B19M is above that of B19" but the difference between them decreases with increasing the $\mathrm{Cu}$ content. At $12.5 \mathrm{at} \% \mathrm{Cu}$, the two phases have similar energy levels. Following the same trend, it may be expected that at $\mathrm{x}>12.5$ at $\% \mathrm{Cu}$ the energy level of $\mathrm{B} 19 \mathrm{M}$ becomes lower than that of B19", i.e. B19M becomes the ground state. This can be the reason of the reported disappearance of B19' (B19") from the thermal transformation sequence of $\mathrm{B} 2 \leftrightarrow \mathrm{B} 19 \leftrightarrow \mathrm{B}^{\prime} 9^{\prime}[\underline{15}]$. 

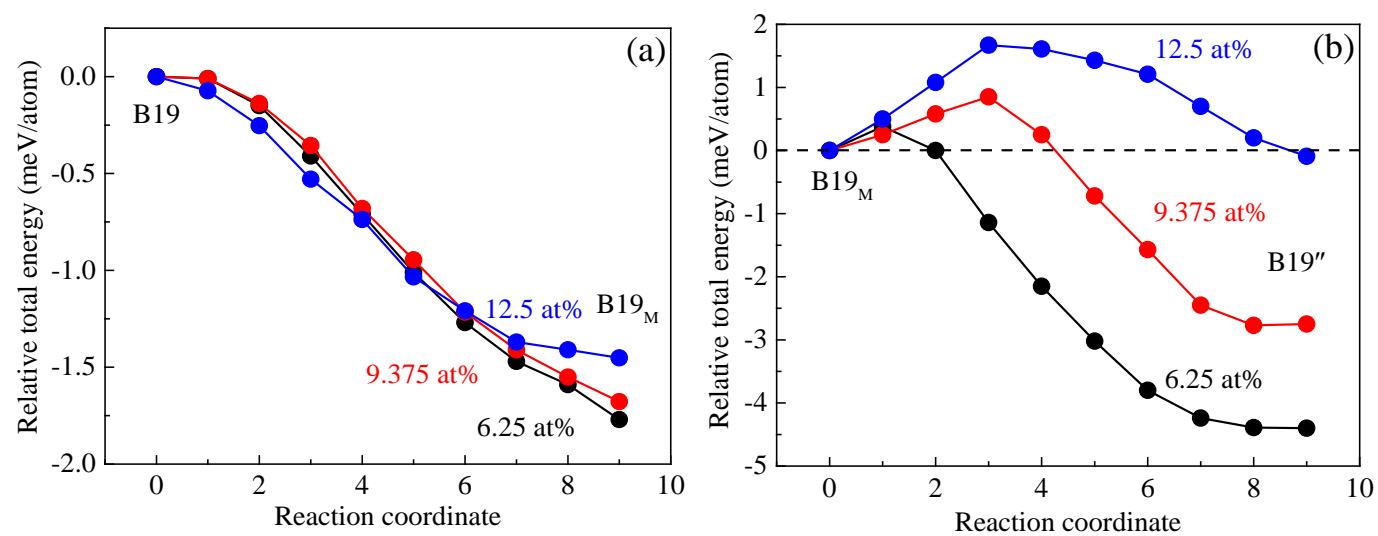

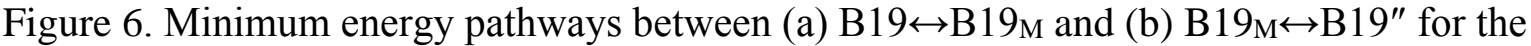
$\mathrm{Ti}\left(\mathrm{Ni}_{50-\mathrm{x}} \mathrm{Cu}\right.$ ) system.

To further assess the stabilities of B19 and В19м phases, phonon dispersions were calculated from the frozen phonon approach using displacements of $0.01 \AA$ in the Phonopy package [41]. Negative frequencies in a phonon dispersion implies dynamic instability of the studied crystal structure. Fig.7 shows the phonon spectra of the phases. Fig.7 (a) shows the phonon dispersion of B19 at 0 at\% Cu content (i.e. equiatomic NiTi alloy system). It is seen that negative phonon frequencies are present near $\Gamma$. This reveals the instability of B19 in the equiatomic NiTi alloy system. Fig. 7(b) shows the phonon dispersion of the B19 phase at the 9.375 at\% Cu content. It is seen that negative frequencies are present which implies that the $\mathrm{Cu}$ addition does not stabilise the B19 phase at $0 \mathrm{~K}$. Fig. 7(c) shows the phonon dispersion of the newly discovered $\mathrm{B} 19 \mathrm{M}$ at $9.375 \mathrm{at} \% \mathrm{Cu}$ content. The positive frequencies reveal the stability of the phase at the studied condition.
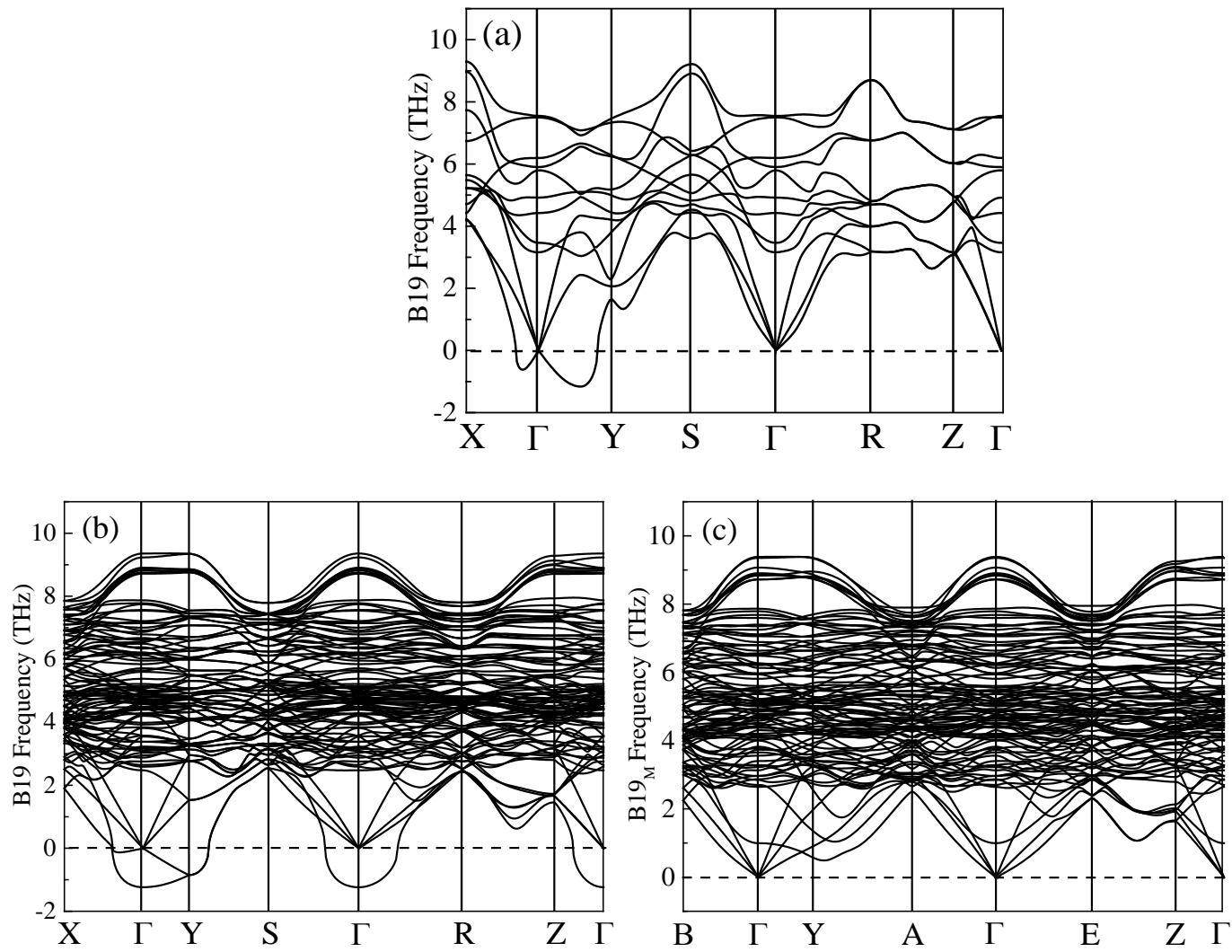
Figure 7. Phonon dispersions of (a) B19 at 0 at\% Cu, (b) B19 at 9.375 at $\% \mathrm{Cu}$ and (c) $\mathrm{B} 19 \mathrm{M}$ at 9.375 at\% $\mathrm{Cu}$ phases at $0 \mathrm{~K}$. Phonons are extracted from simulations using 384-atom super-cells $(2 \times 2 \times 3$ super-cell of $2 \times 2 \times 2$ four-atom primitive unit cells).

Fig. 8 shows the effect of $\mathrm{Cu}$ substitution for $\mathrm{Ni}$ on the unit cell parameters of the possible phases of pseudo-equiatomic $\mathrm{Ti}\left(\mathrm{Ni}_{50-\mathrm{x}} \mathrm{Cu}_{\mathrm{x}}\right)$ alloy system. Fig. 8(a) shows the effect on the unit cell volume of the phases. The unit cell volumes of all the phases increase with increasing $\mathrm{Cu}$ content. The unit cell lattice parameters of B19' at 0 at\% $\mathrm{Cu}$ are experimental values adopted from Kudoh et al. [40], and at 5, 7.5, and 10 at\% Cu are adopted from Nam et al. [30]. The unit cell volume of $\mathrm{B} 19_{\mathrm{M}}$ is close to that of the B19 orthorhombic phase but differs clearly from that of the B19' phase. This indicates that the experimentally observed B19' phase is not a mistaking of the calculated B19M phase. Fig. 8(b) shows the dependences of the calculated lattice parameters of B19 and B19M on Cu content. Lattice constants $a$ and $b$ increase and $c$ decreases with increasing $\mathrm{Cu}$ content for both phases. Apparently, at $12.5 \mathrm{at} \% \mathrm{Cu}$, the unit-cell volume and the lattice parameters $a$ and $c$ of B19 and B19M become very close, but $b$ and the monoclinic angels (Fig. 2) remain still different. Fig. 8(c) shows the effect of Cu content on the lattice parameters of B19" and B19'. The data for B19" are calculated and the data for B19' are experimentally measured. Lattice constants $a$ increases and $b$ decreases with increasing $\mathrm{Cu}$ substitution for Ni for both phases. Lattice constant $c$ increases for B19", but shows no clear trend for B19' with increasing $\mathrm{Cu}$ content.

Comparing the different martensitic phases, B19 and B19M exhibit some partial structural similarities (i.e., similar values of unit cell volume and lattice constants $a$ and $c$ ) whereas B19M and B19" differ from each other in every aspect including the unit cell volume, lattice constants $a$ and $c$ and monoclinic angle. The higher degree of structural similarity between B19 and B19M than between B19 $\mathrm{M}$ and B19" explains the lower critical resistive shear stress required for the $\mathrm{B} 19_{\mathrm{M}} \rightarrow \mathrm{B} 19$ transformation than that for the B19" $\rightarrow$ B19 ${ }_{\mathrm{M}}$ transformation (Figs. 4(a) and (5(a)).

Fig. 8(d) shows the calculated and experimentally measured lattice parameter of B2 phase with increasing $\mathrm{Cu}$ content. The lattice parameter for both cases increases with increasing the $\mathrm{Cu}$ content. The experimentally measured values are higher than those estimated by DFT calculation at all $\mathrm{Cu}$ contents, and the difference between the two increases with increasing the $\mathrm{Cu}$ content. This shows a good agreement between the calculated and measured lattice parameters. 

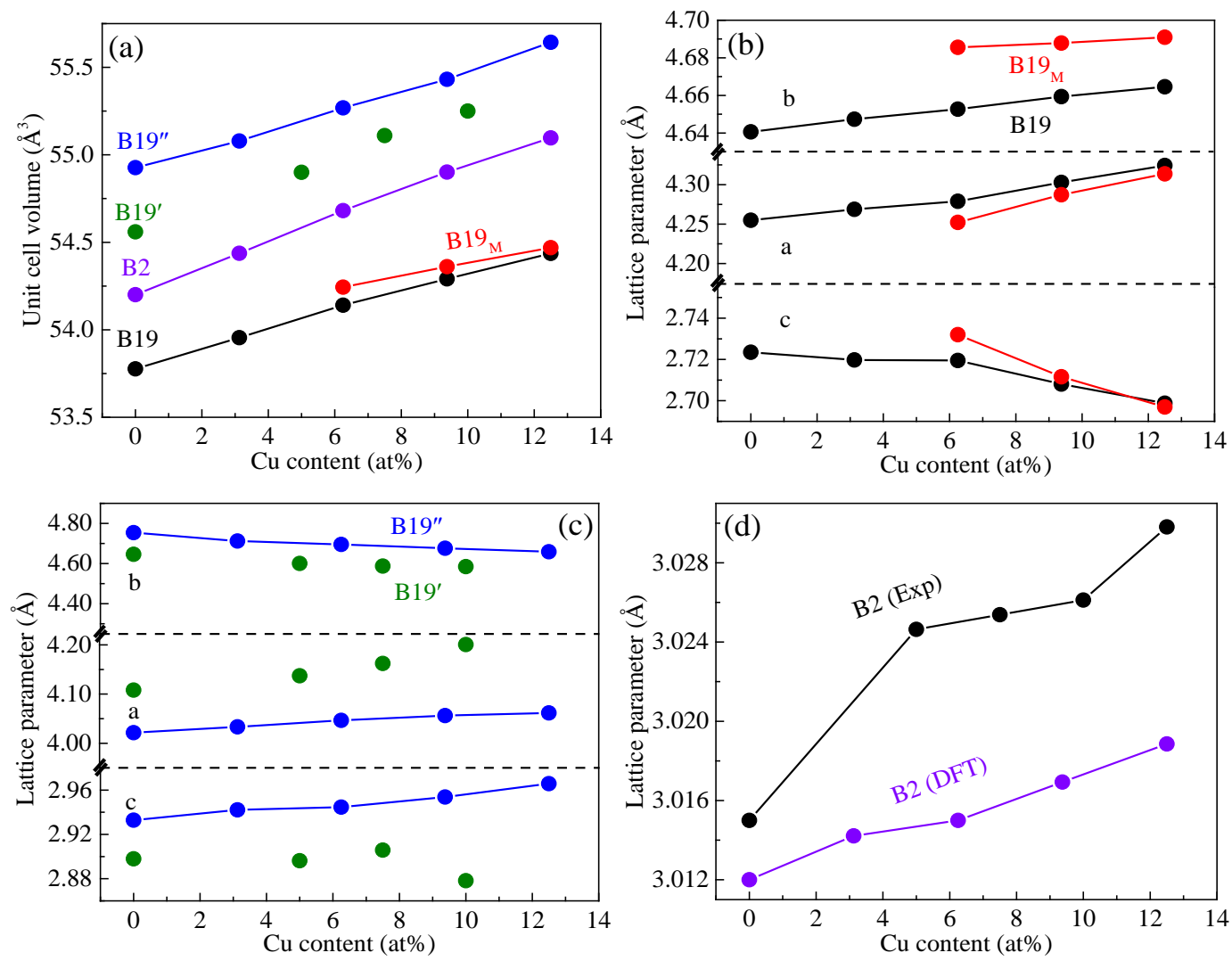

Figure 8. Effect of $\mathrm{Cu}$ content on the unit cell parameters of the parent and the martensitic phases of $\mathrm{Ti}\left(\mathrm{Ni}_{50-\mathrm{x}} \mathrm{Cu}_{\mathrm{x}}\right)$. (a) effect on unit cell volume; (b) effect on lattice constants of B19 and B19M ; (c) effect on lattice constants of B19' and B19"; (d) effect on the lattice parameter of B2. The data for $\mathrm{B} 19^{\prime}$ are from experimental measurements $[\underline{30}, \underline{40}]$.

Fig. 9 shows the effect of $\mathrm{Cu}$ content on the relative total energies of the martensitic phases of $\mathrm{Ti}_{50} \mathrm{Ni}_{50-\mathrm{x}} \mathrm{Cu}_{\mathrm{x}}$ alloy, as computed by means of DFT calculations. The data are relative to the total energy of the B2 phase (the energy difference between the B2 phase and the others). The relative total energy of $\mathrm{B}^{1} 9^{\prime}$ is computed by using the experimentally reported lattice parameters and monoclinic angle of each composition followed by a relaxation of the ionic positions. It is seen that the relative total energy of B19" (and of B19') increases (decrease in absolute value) with increasing $\mathrm{Cu}$ content. This is consistent with the experimental observation that the latent heat of the $\mathrm{B} 2 \leftrightarrow \mathrm{B} 19^{\prime}$ martensitic phase transformation decreases with increasing the $\mathrm{Cu}$ content as measured by differential scanning calorimetry (DSC) [31]. B19" is the ground state at below 12.5 at\% Cu content. For the B19 phase, relative total energy increases moderately with increasing $\mathrm{Cu}$ content up to 6.25 at\% $\mathrm{Cu}$ and remains practically constant at above 6.25 at\% Cu. The relative total energy of B19M is below that of $\mathrm{B} 19$ but above that of B19". It is also apparent that the relative total energy levels of all martensitic phases converge and become comparable with increasing $\mathrm{Cu}$ content to above 12 at\%. From the trend, it is expected that at above 12.5 at\%, В19м becomes the ground state among the martensitic phases. Consequently, the theoretical transformation sequence becomes $\mathrm{B} 2 \leftrightarrow \mathrm{B} 19_{\mathrm{M}}$. This is consistent with the experimental observation of the one-step B2 $\leftrightarrow \mathrm{B} 19$ phase transformation at above 15 at $\% \mathrm{Cu}$ content [32]. 


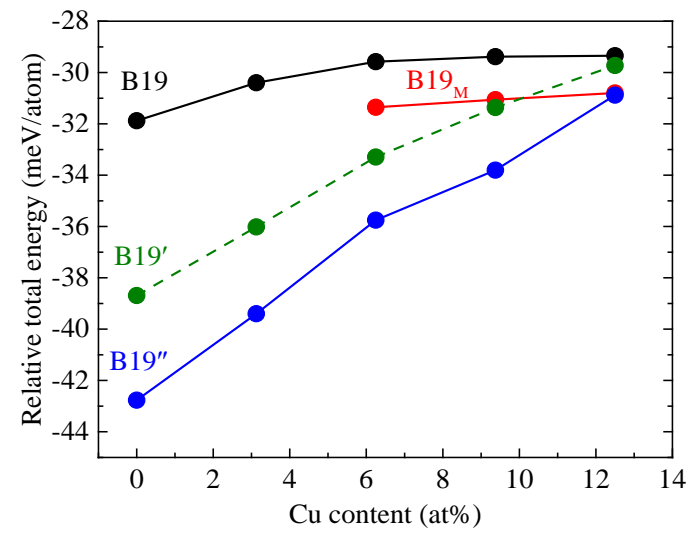

Figure 9. Effect of $\mathrm{Cu}$ content on the total energies of the martensitic phases relative to that of the B2 parent phase in $\mathrm{Ti}_{50} \mathrm{Ni}_{50-\mathrm{x}} \mathrm{Cu}_{\mathrm{x}}$ alloy. The data for $\mathrm{B} 19^{\prime}$ are DFT calculated using the experimentally measured unit cell parameters $[\underline{30}, \underline{40}]$.

\section{Conclusions}

This study investigated the effect of $\mathrm{Cu}$ substitution for $\mathrm{Ni}$ in pseudo-equiatomic $\mathrm{Ti}\left(\mathrm{Ni} \mathrm{F}_{50-\mathrm{x}} \mathrm{Cu}\right.$ $x$ ) alloy on the structures and stabilities of the various martensitic phases. The main findings are summarised below:

(1) A new monoclinic phase (B19M) is identified to form at $\mathrm{x} \geq 9.25$ at $\%$. This phase is distinctively different from the known orthorhombic B19 and monoclinic B19' phases.

(2) The monoclinic angles of the B19 $\mathrm{M}, \mathrm{B} 19^{\prime}$, and B19" structures decrease with increasing the $\mathrm{Cu}$ content in the pseudo-equiatomic $\mathrm{Ti}\left(\mathrm{Ni}_{50-\mathrm{x}} \mathrm{Cu}_{\mathrm{x}}\right)$ system.

(3) The B19 $\rightarrow \mathrm{B} 19 \mathrm{M}$ transformation has a barrier-less minimum energy pathway, i.e., the B19 phase is unstable under no external influences (e.g., shear stress) and the transformation is spontaneous.

(4) The B19 $\rightarrow$ B19" transformation is associated with an energy barrier in the minimum energy pathway and the magnitude of the barrier increases with increasing the $\mathrm{Cu}$ content. The presence of the energy barrier indicates that $\mathrm{B} 19_{\mathrm{M}}$ is a stable phase.

(5) In the pseudo-equiatomic $\mathrm{Ti}\left(\mathrm{Ni}_{50-\mathrm{x}} \mathrm{Cu}_{\mathrm{x}}\right)$ system, at $\mathrm{x} \leq 12.5$ at $\% \mathrm{~B} 19^{\prime \prime}$ is the ground state and the theoretical transformation pathway is $\mathrm{B} 2 \leftrightarrow \mathrm{B} 19_{\mathrm{M}} \leftrightarrow \mathrm{B} 19^{\prime \prime}$. At $\mathrm{x}>12.5$ at $\%$, it is predicted that the $\mathrm{B} 19 \mathrm{M}$ becomes the ground state and the theoretical transformation pathway changes to $\mathrm{B} 2 \leftrightarrow \mathrm{B} 19 \mathrm{M}$.

The evidence collected in this study also enables the following hypotheses:

(6) The experimentally observed B19' typically with a monoclinic angle of $97.8^{\circ}$ in binary NiTi (i.e., at $\mathrm{x}=0 \mathrm{at} \% \mathrm{Cu}$ ) is in fact a distorted B19" phase with a reduced monoclinic angle from $101.6^{\circ}$. This can be rationalised by the inherent opposing shear stress associated with the monoclinic lattice distortion of the B19" (and B19') phase. In this regard, it is in essence not a thermodynamically stable phase and its monoclinic angle is expected to change depending on the actual magnitude of the opposing shear stress upon each variant.

(7) By the same argument, the experimentally observed orthorhombic B19 phase reported in the literature for $\mathrm{Ti}(\mathrm{Ni}, \mathrm{Cu})$ alloys is in fact a metastable phase formed from $\mathrm{B} 19_{\mathrm{M}}$ under the influence of an opposing shear stress. This can also be rationalised by the inherent opposing shear stress associated with the monoclinic lattice distortion of the В19м phase. 


\section{Acknowledgement}

We wish to acknowledge the financial support to this work from the Australian Research Council in grant 180101955 . This work was supported by resources provided by the Pawsey Supercomputing Centre with funding from the Australian Government and the Government of Western Australia, and by National Computational Infrastructure.

\section{References}

[1] K. Otsuka, X. Ren, Physical metallurgy of Ti-Ni-based shape memory alloys, Progress in materials science 50(5) (2005) 511-678.

[2] Q. Meng, Z. Wu, R. Bakhtiari, B.S. Shariat, H. Yang, Y. Liu, T.-h. Nam, A unique "fishtaillike” four-way shape memory effect of compositionally graded NiTi, Scripta Materialia 127 (2017) 84-87.

[3] Q. Meng, Z. Wu, R. Bakhtiari, J. Zhang, H. Yang, Y. Liu, Stress serration and arch-shaped Lüders stress plateau behaviour of $\mathrm{Ti}-50.8$ at\% $\mathrm{Ni}$ wire prepared by selective electrical resistance over-aging, Smart Materials and Structures 25(11) (2016) 115035.

[4] F. Butera, A. Coda, G. Vergani, S.G. SpA, Shape memory actuators for automotive applications, Nanotec IT newsletter. Roma: AIRI/nanotec IT (2007) 12-6.

[5] C. Bil, K. Massey, E.J. Abdullah, Wing morphing control with shape memory alloy actuators, Journal of Intelligent Material Systems and Structures 24(7) (2013) 879-898.

[6] M. Kohl, Shape memory microactuators, Springer Science \& Business Media2013.

[7] J. Ruiz-del-Solar, E. Chown, P.G. Plöger, RoboCup 2010: Robot Soccer World Cup XIV, Springer Science \& Business Media2011.

[8] L. Petrini, F. Migliavacca, Biomedical applications of shape memory alloys, Journal of Metallurgy 2011 (2011).

[9] S. Hao, L. Cui, D. Jiang, X. Han, Y. Ren, J. Jiang, Y. Liu, Z. Liu, S. Mao, Y. Wang, A transforming metal nanocomposite with large elastic strain, low modulus, and high strength, Science 339(6124) (2013) 1191-1194.

[10] B.S. Shariat, Q. Meng, A.S. Mahmud, Z. Wu, R. Bakhtiari, J. Zhang, F. Motazedian, H. Yang, G. Rio, T.-h. Nam, Experiments on deformation behaviour of functionally graded NiTi structures, Data in Brief 13 (2017) 562-568.

[11] R. Bakhtiari, B.S. Shariat, F. Motazedian, Z. Wu, J. Zhang, H. Yang, Y. Liu, Complex transformation field created by geometrical gradient design of NiTi shape memory alloy, Functional Materials Letters 10(01) (2017) 1740011.

[12] B.S. Shariat, Q. Meng, A.S. Mahmud, Z. Wu, R. Bakhtiari, J. Zhang, F. Motazedian, H. Yang, G. Rio, T.-h. Nam, Functionally graded shape memory alloys: Design, fabrication and experimental evaluation, Materials \& Design 124 (2017) 225-237. 
[13] B.S. Shariat, R. Bakhtiari, Y. Liu, Nonuniform transformation behaviour of NiTi in a discrete geometrical gradient design, Journal of Alloys and Compounds 774 (2018) 1260-1266.

[14] Y. Furuya, Design and material evaluation of shape memory composites, Journal of intelligent material systems and structures 7(3) (1996) 321-330.

[15] T.H. Nam, T. Saburi, K.i. Shimizu, Cu-content dependence of shape memory characteristics in Ti-Ni-Cu alloys, Materials Transactions, JIM 31(11) (1990) 959-967.

[16] H. Shi, J. Frenzel, G. Martinez, S. Van Rompaey, A. Bakulin, S. Kulkova, S. Van Aert, D. Schryvers, Site occupation of $\mathrm{Nb}$ atoms in ternary Ni-Ti-Nb shape memory alloys, Acta Materialia 74 (2014) 85-95.

[17] J. Frenzel, A. Wieczorek, I. Opahle, B. Maaß, R. Drautz, G. Eggeler, On the effect of alloy composition on martensite start temperatures and latent heats in Ni-Ti-based shape memory alloys, Acta Materialia 90 (2015) 213-231.

[18] S. Saghaian, H. Karaca, M. Souri, A. Turabi, R. Noebe, Tensile shape memory behavior of Ni 50.3 Ti 29.7 Hf 20 high temperature shape memory alloys, Materials \& Design 101 (2016) 340-345.

[19] A.C. Coppa, M. Kapoor, R. Noebe, G.B. Thompson, The compositional stability of the Pphase in Ni-Ti-Pd shape memory alloys, Intermetallics 67 (2015) 56-62.

[20] L. Gou, Y. Liu, T.Y. Ng, An investigation on the crystal structures of Ti 50 Ni 50- x Cu $\mathrm{x}$ shape memory alloys based on density functional theory calculations, Intermetallics 53 (2014) 20-25.

[21] D. Dye, Shape memory alloys: Towards practical actuators, Nature materials 14(8) (2015) 760-761.

[22] C. Chluba, W. Ge, R.L. de Miranda, J. Strobel, L. Kienle, E. Quandt, M. Wuttig, Ultralowfatigue shape memory alloy films, Science 348(6238) (2015) 1004-1007.

[23] X. Huang, G.J. Ackland, K.M. Rabe, Crystal structures and shape-memory behaviour of NiTi, Nature materials 2(5) (2003) 307-311.

[24] K.G. Vishnu, A. Strachan, Phase stability and transformations in NiTi from density functional theory calculations, Acta Materialia 58(3) (2010) 745-752.

[25] M.-X. Wagner, W. Windl, Lattice stability, elastic constants and macroscopic moduli of NiTi martensites from first principles, Acta Materialia 56(20) (2008) 6232-6245.

[26] D. Holec, M. Friák, A. Dlouhý, J. Neugebauer, Ab initio study of pressure stabilized NiTi allotropes: Pressure-induced transformations and hysteresis loops, Physical Review B 84(22) (2011) 224119.

[27] S.R. Bakhtiari, J.Z. Liu, B.S. Shariat, H. Yang, Y. Liu, Role of hydrostatic pressure on the phase stability, the ground state, and the transformation pathways of NiTi alloy, Scripta Materialia 151 (2018) 57-60. 
[28] W.-S. Ko, Temperature dependence of NiTi martensite structures: Density functional theory calculations, Scripta Materialia 154 (2018) 134-138.

[29] S. Kibey, H. Sehitoglu, D. Johnson, Energy landscape for martensitic phase transformation in shape memory NiTi, Acta Materialia 57(5) (2009) 1624-1629.

[30] T.H. Nam, T. Saburi, Y. Nakata, K.i. Shimizu, Shape memory characteristics and lattice deformation in Ti-Ni-Cu alloys, Materials Transactions, JIM 31(12) (1990) 1050-1056.

[31] W. Tang, R. Sandström, Z. Wei, S. Miyazaki, Experimental investigation and thermodynamic calculation of the Ti-Ni-Cu shape memory alloys, Metallurgical and materials transactions A 31(10) (2000) 2423-2430.

[32] P. Potapov, A. Shelyakov, D. Schryvers, On the crystal structure of TiNi-Cu martensite, Scripta materialia 44(1) (2001) 1-7.

[33] T. Ohba, T. Taniwaki, H. Miyamoto, K. Otsuka, K. Kato, In situ observations of martensitic transformations in Ti50Ni34Cu16 alloy by synchrotron radiation, Materials Science and Engineering: A 438 (2006) 480-484.

[34] T. Goryczka, M. Karolus, P. Ochin, H. Morawiec, Martensites in NiTi and NiTiCu alloysStructure of melt spun Ni25Ti50Cu25 ribbons studied by X-ray diffraction, Journal de Physique-Colloques 11(8) (2001) 345-350.

[35] G. Kresse, D. Joubert, From ultrasoft pseudopotentials to the projector augmented-wave method, Physical Review B 59(3) (1999) 1758.

[36] J.P. Perdew, K. Burke, M. Ernzerhof, Generalized gradient approximation made simple, Physical review letters 77(18) (1996) 3865.

[37] P.E. Blöchl, Projector augmented-wave method, Physical review B 50(24) (1994) 17953.

[38] D. Sheppard, P. Xiao, W. Chemelewski, D.D. Johnson, G. Henkelman, A generalized solid-state nudged elastic band method, The Journal of chemical physics 136(7) (2012) 074103.

[39] A. Zunger, S.-H. Wei, L. Ferreira, J.E. Bernard, Special quasirandom structures, Physical Review Letters 65(3) (1990) 353.

[40] Y. Kudoh, M. Tokonami, S. Miyazaki, K. Otsuka, Crystal structure of the martensite in Ti-49.2 at.\% Ni alloy analyzed by the single crystal X-ray diffraction method, Acta Metallurgica 33(11) (1985) 2049-2056.

[41] A. Togo, I. Tanaka, First principles phonon calculations in materials science, Scripta Materialia 108 (2015) 1-5. 